\title{
OCORRÊNCIA DE DINOFLAGELADOS DO GÊNERO Dinophysis (ENRENBERG, 1839) NA ENSEADA DE CABEÇUDAS (VERÃO E OUTONO DE 1999)
}

\author{
SCHMITT, F. \& L.A. PROENÇA \\ CTTMar - UNIVALI \\ Rua Uruguai, 458 - Cx. P. 360, CEP: 88302-202, Itajaí/SC \\ e-mail: proenca@cttmar.univali.br / fabricio.schmitt@zipmail.com.br
}

\begin{abstract}
RESUMO
Os dinoflagelados compõem um dos principais grupos do plâncton marinho, incluindo organismos autotróficos e heterotróficos, onde aproximadamente 40 são produtores de ficotoxinas. As ficotoxinas produzidas por dinoflagelados podem ser classificadas de acordo com o grupo químico em: envenenamento diarréico de marisco (DSP), envenenamento paralisante de marisco (PSP), envenenamento neurológico de marisco (NSP) e ciguatera. Os dinoflagelados produtores de toxinas DSP restringem-se aos gêneros Dinophysis e Prorocentrum, e incluem: $D$. acuminata, $D$. acuta, $D$. fortii, $D$. norvegica, $D$. mitra, $D$. rotundata, $D$. tripos, $D$. sacculus, $D$. skagii e $P$. lima. Com o objetivo de conhecer a ocorrência do gênero Dinophysis na praia de Cabeçudas, no município de Itajaí/SC, amostras sub-superficiais da coluna d'água e de arrasto com rede de plâncton foram coletadas semanalmente entre os dias 6/1/1999 e 29/6/1999, num total de 24 amostras. Foram também realizadas medições da salinidade, temperatura, nutrientes inorgânicos dissolvidos e da biomassa do fitoplâncton. A temperatura da água variou entre $29^{\circ} \mathrm{C} \mathrm{e} 17^{\circ} \mathrm{C}$, enquanto a salinidade entre 36 e 25 . O máximo e mínimo de clorofila a foi de $3,97 \mu \mathrm{g} . \mathrm{I}^{-1} \mathrm{e}$ 0,83 $\mu \mathrm{g} . \mathrm{I}^{-1}$, respectivamente. Entre os nutrientes inorgânicos, amônio variou de $12,65 \mu \mathrm{mol} . \mathrm{I}^{-1}$ à $6,85 \mu \mathrm{mol} . \mathrm{I}^{-1}$, nitrato de $6,34 \mu \mathrm{mol}^{. \mathrm{I}^{-1}}$ à $0,76 \mu \mathrm{mol} . \mathrm{I}^{-1}$, silício de $54,12 \mu \mathrm{mol} . \mathrm{I}^{-1}$ à $5,75 \mu \mathrm{mol} . \mathrm{I}^{-1}$ e fosfato de $0,82 \mu \mathrm{mol} . \mathrm{I}^{-1}$ à $0,09 \mu \mathrm{mol}^{-I^{-1}}$. A ocorrência de Dinophysis foi restrita a: $D$. acuminata, $D$. rotundata, $D$. caudata e $D$. spp. A abundância em geral de Dinophysis foi baixa, sendo encontrado o máximo de 80 cel. $^{-1}{ }^{-1}$ para D. caudata e $D$. rotundata, nos dias 2/2/99 e 23/3/99, respectivamente. A análise estatística não apresentou correlação significativa entre o aparecimento de Dinophysis spp. e as variáveis analisadas. Além das espécies de Dinophysis, outros microrganismos produtores de toxinas lipossolúveis ou paralisantes também foram detectados. Estes valores indicam a ausência de DSP na região, durante o período amostrado, considerando a abundância de $100 \mathrm{cel}^{\mathrm{I}} \mathrm{I}^{-1}$, adotada por certos países como limite superior para a extração segura de moluscos.
\end{abstract}

Palavras Chave: Dinophysis spp., Cabeçudas, nutrientes, DSP

\section{ABSTRACT \\ OCCURRENCE OF THE DINOFLAGELLATE Dinophysis(ENRENBERG, 1839) AT CABEÇUDAS BIGHT (SUMMER TO AUTUMN, 1999)}

\footnotetext{
Dinoflagellate is a main group in the marine plankton and includes both, autotrophic and heterotrophic organism. Some species are known to produce different sort of toxins which can harm also human beings. Such phycotoxins are classified according to its chemical characteristics or simptomatology into 4 groups: diarhetic shellfish poisoning (DSP), paralytic shellfish poisoning (PSP), neurotoxic shellfish poisoning (NSP) and ciguatera. DSP producing dinoflagellates are distributed into 2 genera, Prorocentrum and Dinophysis, including P. lima, D. acuminata, D. acuta, $D$. fortii, $D$. norvergica, $D$. mitra, $D$. rotundata, $D$. tripos, $D$. sacculus, $D$. skagii and others. Several of these species are known to occur in Brazil and DSP has been recently detected in the
} 
littoral of Santa Catarina. This paper aims to investigate the occurrence of Dinophysis at Cabeçudas beach, Itajaí, Santa Catarina. Water column samples were collected weekly from 6/01/1999 to 29/06/1999. Samples for microscopic examination were collected from the sub surface and from the water column by net tolls. Salinity, temperature, chlorophyll a and inorganic nutrients were also analyzed. Water temperature ranged from 17 to $29^{\circ} \mathrm{C}$ and salinity from 25 to 36 . Maximum and

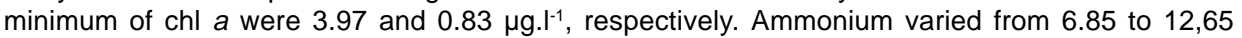
$\mu \mathrm{mol} . \mathrm{I}^{-1}$, while nitrate plus nitrite from 0.76 to $6.34 \mu \mathrm{mol}^{. \mathrm{I}^{-1}}$; silicate from 5.75 to $54.12 \mu \mathrm{mol}^{-\mathrm{I}^{-1}}$ and phosphate from 0.09 to $0,82 \mu \mathrm{mol}^{-l^{-1}}$. Just 3 species of Dinophysis were observed: $D$. acuminata, $D$. rotundata e $D$. caudata and one Dinophysis sp. non identified. In general the abundance was

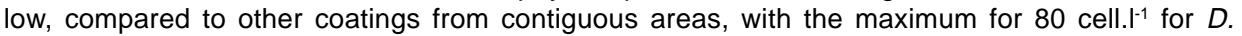
caudata and $D$. rotundata, found in 2/02/1999 and 23/03/1991, respectively. No significant correlation among the occurrence of Dinophysis and the other variables were observed. Apart from Dinophysis, other toxic species were also observed. These results indicate that, at least during the sampled period, the abundance of DSP producers was low in the region, indicating a safe mussel extraction, considering the superior limit of 100 cell.. $^{-1}$, as adopted in some countries

Keywords: Dinophysis spp., Cabeçudas, nutrients, DSP

\section{INTRODUÇÃO}

Dentre as intoxicações causadas por organismos marinhos, destacam-se aquelas originadas pelo fenômeno conhecido popularmente como "maré vermelha". Maré vermelha é o nome comum dado ao fenômeno de coloração das águas produzido por algumas espécies de organismos fitoplanctônicos. Embora a coloração seja óbvia, algumas espécies que produzem toxinas não colorem a água podendo ser nocivas mesmo em baixas abundâncias. Sabendo das limitações do termo "maré vermelha", ultimamente tem-se usado a expressão "Florações de Algas Nocivas (FAN)" (IOC, 1995). Os fenômenos de FAN são acumulações ou crescimento populacional excessivo de organismos fitoplanctônicos que podem chegar até bilhões de células por litro (Carreto, 1991). Margalef (1983) relaciona a ocorrência de florações dos diferentes organismos à alteração na disponibilidade de luz e nutrientes condicionados pela turbulência do meio.

Atualmente são conhecidas cerca de 5000 espécies de microalgas, sendo que destas, 300 formam florações e 40 são potencialmente produtoras de toxinas (Hallegraeff et al., 1995). Existem três tipos de efeitos nocivos de algas: 1) aqueles gerados por espécies não tóxicas que após uma floração podem ocasionar anoxia na coluna d'água, levando a morte peixes e invertebrados; 2) gerados por espécies produtoras de ficotoxinas as quais podem chegar ao homem via cadeia trófica; e 3) aqueles gerados por espécies não tóxicas ao homem, mas nocivas a peixes ou invertebrados por danos às brânquias ou obstrução do sistema de filtração. Dentre as espécies que produzem ficotoxinas podemos citar diferentes grupos taxonômicos como as diatomáceas, cianobactérias e os dinoflagelados (Larsen \& Moestrup, 1989; Hallegraeff et al., 1995).

As ficotoxinas podem ser classificadas de acordo com o grupo químico ou os sintomas produzidos em: envenenamento diarréico de marisco (DSP), envenenamento neurológico de marisco (NSP), envenenamento paralisante de marisco (PSP), envenenamento amnésico de marisco (ASP), veneno de cianobactérias (VC) e ciguatera. Os dinoflagelados, que são os principais produtores de toxinas de DSP, NSP, PSP e ciguatera, formam um grupo de microrganismos unicelulares, caracterizados pela presença de dois flagelos dinocontes. São geralmente autotróficos, porém podem apresentar auxotrofia ou heterotrofia. Estão distribuídos em regiões costeiras e oceânicas, com representantes tanto no bentos como na coluna d'água.

Nas últimas décadas tem-se verificado um aumento aparente na ocorrência de intoxicações relacionadas a ficotoxinas a nível global, incluindo DSP. Este aumento aparente 
pode ser atribuído a diversas causas, sendo as principais: a) eutrofização das águas costeiras; b) controle de qualidade do produto consumido; c) dispersão de esporos por água de lastro de navios transoceânicos; d) dispersão por correntes; e e) aumento de áreas de cultivo ou exploração de moluscos (Hallegraeff, 1993). O primeiro caso registrado de contaminação por DSP em humanos, ocorreu na Holanda em 1960 e foi associado ao consumo de mexilhões que haviam filtrado dinoflagelados (Kat, 1979). Apesar desta ocorrência, o DSP só foi descoberto em 1976, no Japão, após a intoxicação de diversos consumidores de mexilhão (Yasumoto et al., 1978). Amostras destes moluscos apresentaram toxicidade por meio de um bioensaio com camundongos utilizando-se um extrato orgânico. Posteriormente, a toxina ácido ocadaico (AO) foi isolada e classificada como a principal responsável pelas intoxicações. Sua ocorrência foi associada a um dinoflagelado do gênero Dinophysis. O efeito tóxico do $\mathrm{AO}$ em humanos está relacionado a distúrbios gastrointestinais, incluindo calafrios, diarréia, náusea e vômitos.

Os microrganismos produtores de toxinas de DSP restringem-se aos gêneros Dinophysis e Prorocentrum, e incluem $D$. acuminata, $D$. acuta, $D$. fortii, $D$. norvegica, $D$. mitra, $D$. rotundata, $D$. tripos, $D$. sacculus, $D$. skagii e P. lima, (Larsen \& Moestrup, 1989; Johansson et al., 1996; 1996; Maestrini, 1998). No entanto, acredita-se que todas as espécies de Dinophysis sejam tóxicas. Contudo, a confirmação da toxicidade das espécies de Dinophysis é difícil, uma vez que estes microrganismos não podem ser mantidos em cultivo.

Embora existam muitos estudos sobre a ecologia e distribuição do fitoplâncton (Brandini et al., 1997), pouco se sabe sobre microrganismos produtores de toxinas e seus efeitos no Brasil. Até o momento, apenas uma ocorrência de intoxicação de consumidores de mexilhão foi associada a DSP. Este caso ocorreu em Florianópolis em 1991, quando várias pessoas apresentaram os sintomas de DSP após a ingestão de moluscos. Amostras da água coletadas na época evidenciaram a presença de Dinophysis em abundância. Bioensaios realizados pelo Instituto Adolfo Lutz (São Paulo) indicaram toxicidade nos moluscos consumidos (Zenebon \& Pregnolato, 1992). O AO foi detectado, pela primeira vez, em amostra no cultivo da praia de Laranjeiras (SC), (Proença et al., 1996), e mais tarde na Enseada de Armação do Itapocoróy, em Penha/SC (Proença et al., 1998). Posteriormente, o AO foi detectado no microrganismo Dinophysis acuminata, (Proença et al., 1998). No entanto, os poucos trabalhos de taxonomia realizados no litoral de Santa Catarina (Cardoso, 1993; Rörig et al., 1998), indicam a presença de várias espécies de Dinophysis. Os trabalhos de identificação desenvolvidos em áreas de cultivo apontam a ocorrência de ao menos seis espécies de Dinophysis: D. caudata, D. ovum, $D$. acuminata, $D$. tripos, $D$. acuta, e $D$. rotundata (Rörig, L. R., CTTMar/UNIVALI, com. pes.). A ocorrência de espécies tóxicas é utilizada como uma ferramenta complementar no monitoramento dos níveis de toxinas em moluscos cultivados (Andersen, 1996). Alguns países, por exemplo, adotam a contagem de

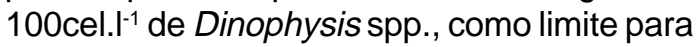
o consumo de mexilhões na área de cultivo. Neste contexto, o presente trabalho visou determinar a ocorrência de dinoflagelados do gênero Dinophysis na praia de Cabeçudas e suas interações com fatores abióticos.

\section{METODOLOGIA}

O presente estudo foi desenvolvido na praia de Cabeçudas, localizada no município de Itajaí/SC, a aproximadamente $3 \mathrm{~km}$ ao sul da foz do Rio Itajaí-Açu (Figura 1). A praia tem aproximadamente $900 \mathrm{~m}$ e é influenciada diretamente pela descarga do rio Itajaí-Açu. O local de coleta foi situado na posição $2655^{\prime} 40$ "S e 48 37' 40"W, junto ao trapiche do late Club, com profundidade de aproximadamente $2 \mathrm{~m}$, sendo influenciado ocasionalmente pela presença de ondas. Para observar a ocorrência de Dinophysis spp. na praia de Cabeçudas, foram 
realizadas amostragens semanais, no período entre 6/1/99 à 29/6/99, totalizando 24 amostras (Tabela 1). A coleta qualitativa foi feita junto a superfície da coluna d'água, com auxílio

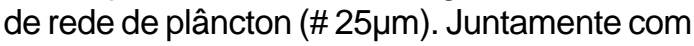
a amostragem de rede, foram coletadas amostras quantitativas de sub-superfície, com auxílio de um balde. As amostras quantitativas e qualitativas, foram fixadas em lugol (1\%) e formol (4\%), respectivamente, no local de coleta. A quantificação de Dinophysis spp. foi feita pelo método de sedimentação (UTERMÖHL, 1958), em microscópio invertido marca Olympus modelo IX-50 S8F. Subamostras fixadas em lugol foram acondicionadas em câmaras de sedimentação de $50 \mathrm{ml}$, por um período de 24h. Dada a pouca abundância dos organismos estudados, as contagens foram feitas em toda a lâmina. Além das espécies de Dinophysis foram incluídas nas contagens os dinoflagelados do gênero Prorocentrum e Ceratium. Conjuntamente com as amostras de

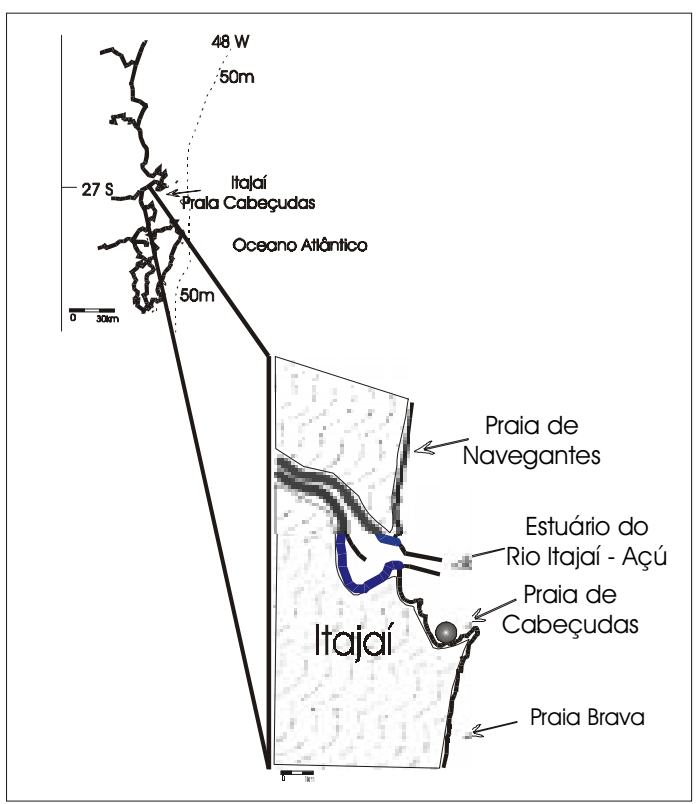

Figura 1 - Área de estudo, mostrando a praia de Cabeçudas, o ponto amostral junto ao trapiche do late Club

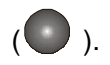

plâncton, foi realizado um acompanhamento das concentrações de nutrientes inorgânicos dissolvidos: fosfato, amônio, nitrato e silicato. Neste trabalho, o valor de nitrato foi considerado o somatório das concentrações de nitrato e nitrito. Após a coleta, as amostras de água, foram trazidas para laboratório e filtradas em filtro de fibra de vidro tipo Whatman GF/F (\#0,7 $\mu \mathrm{m})$. O espectrofotômetro utilizado para a verificação da concentração dos nutrientes foi da marca Shimadzu, modelo UV-V160A com duplo feixe. A quantificação foi realizada pelos métodos tradicionais de Mullin \& Riley (1955), Wood et al. (1967), Strickland \& Parsons (1972), e Murphy \& Riley (1962), descritos em Baumgarten et al. 1996, para silício, nitrato, amônio e fosfato, respectivamente. A biomassa do fitoplâncton foi estimada pela quantificação da clorofila a, pelo método de cromatografia líquida de alta eficiência (CLAE), adaptado de Mantoura et al. (1997). As amostras da coluna d'água foram filtradas em filtro de fibra de vidro Whatman GF/F (\#0,7 $\mu \mathrm{m})$ em sistema à vácuo. O filtro foi congelado até posterior análise. Ao filtro foram adicionados $4 \mathrm{ml}$ de metanol e as células destruídas em sonda de ultra-som marca Sonics \& Materials Inc, modelo VC100. A temperatura do extrato durante a sonicação foi mantida constante com imersão do frasco em água com gelo. $\mathrm{O}$ extrato foi centrifugado em centrífuga Eppendorf, modelo Centrifuge 5410, para clarificação. O sistema de CLAE utilizado foi do tipo ShimadzuÒ LC-10A composto de: sistema de degassificação por borbulhamento de hélio, bomba de alta pressão LC10-AD, detector de fluorescência RF-551, um injetor Rheodyne $7725 \mathrm{i}$ e um integrador de sinais CR6A. A cromatografia foi realizada em uma coluna SHIM-PACK, $(4,6 \mathrm{~mm}$ de diâmetro interno e $40 \mathrm{~mm}$ de comprimento) empacotada com partículas ODS de $3 \mu \mathrm{m}$ de diâmetro. A $160 \mu \mathrm{l}$ da amostra clarificada, foram adicionados $40 \mu \mathrm{l}$ de acetato de amônia. Esta amostra foi eluída em um sistema isocrático, constituído de metanol e acetona, numa proporção de $8: 2$, com velocidade de fluxo de $0,7 \mathrm{ml}$ por minuto, durante 4 minutos. $O$ registro de 
fluorescência foi obtido com $435 \mathrm{~nm}$ de excitação e $660 \mathrm{~nm}$ de emissão. Os solventes empregados foram de grau HPLC e a água filtrada em sistema Milli-Q (Whaters Co.). O acetato de amônio foi de pureza PA. A calibração foi realizada com padrão de cla a pura (Sigma). A cada dez amostras analisadas, foram injetados $80 \mu l$ de padrão de cla $a$. O cálculo da concentração de cla a nas amostras foi obtido através do fator $(f)$, igual a concentração de cla a injetada no padrão, dividida pela sua respectiva área. $\mathrm{O}$ valor de $f$ calculado foi de
$3,01663 \times 10^{-8} \mu \mathrm{g} . \mathrm{s} . \mu \mathrm{v}^{-1}$. A área da amostra foi multiplicado pelo $f$ obtendo-se a quantidade de cla a injetada. Para quantificar a cla a em litro, foi aplicada a equação abaixo,

$$
\text { cla }=f * A a *(\mathrm{Ve} / \mathrm{Vi}) *(\mathrm{Cd} / \mathrm{Vf})
$$

onde: cla $=$ concentração de cla $a$ em $\mu g . I^{-1} ; f$ = fator de calibração (microgramas por segundo por microvolt $\mu$ g.s. $\left.\mu v^{-1}\right) ; A a=$ área da amostra em $\mu v \cdot s^{-1} ; \mathrm{Ve}=$ volume da extração em $\mu \mathrm{l}$ $(4000 \mu \mathrm{l}) ; \mathrm{Vi}=$ volume injetado em $\mu \mathrm{l}(80 \mu \mathrm{l}) ; \mathrm{Cd}$

Tabela 1 - Dados obtidos na praia de Cabeçudas entre os dias 6/1/1999 e 29/6/1999. Temperatura $\left({ }^{\circ} \mathrm{C}\right)$, salinidade (sal), descarga em $\mathrm{m}^{3} \cdot \mathrm{s}^{-1}(\mathrm{Q})$, amônia $(\mathrm{A})$, silício (S), fosfato $(\mathrm{F})$ e nitrato + nitrito $(\mathrm{N})$ em $\mu \mathrm{mol.} \mathrm{I}^{-1}$, clorofila a em $\mu \mathrm{g} . \mathrm{I}^{-1}$ (Cla a), Dinophysis acuminata (D.a.), D. rotundata (D.r.), D. caudata (D.c.), Dinophysis spp (D.spp.), Ceratium furca (C.f.), Ceratium spp (C.spp.), Prorocentrum gracile (P.g.), P. micans (P.m.), Prorocentrum spp (P.spp) em cel.. ${ }^{-1}$, máximo (Max), mínimo (Min), média (x), desvio padrão (s), coeficiente de variação em \% (CV), número de amostras $(\mathrm{N})$, erro padrão (EP).

\begin{tabular}{|c|c|c|c|c|c|c|c|c|c|c|c|c|c|c|c|c|c|}
\hline Data & ${ }^{\circ} \mathrm{C}$ & sal & Q & A & $S$ & $\mathrm{~F}$ & $\mathrm{~N}$ & Cla a & D.a & D. $r$ & D. $C$ & D. spp. & C. $f$. & C. spp. & P. g. & P. m. & P. spp. \\
\hline $6 / 1$ & 25 & 32 & 286,4 & & 16,47 & 0,29 & 0,96 & 2,41 & 20 & 0 & 40 & 0 & 0 & 0 & 200 & 20 & 0 \\
\hline $12 / 1$ & 25 & 35 & 155,7 & 9,18 & 6,42 & 0,44 & 1,32 & 3,98 & 0 & 0 & 0 & 0 & 0 & 0 & 100 & 0 & 0 \\
\hline $19 / 1$ & 25 & 34 & 166,9 & 7,95 & 11,48 & 0,51 & 1,28 & 0,88 & 20 & 0 & 0 & 0 & 0 & 0 & 140 & 20 & 20 \\
\hline $26 / 1$ & 26 & 27 & 314,5 & 11,62 & 48,76 & 0,29 & 6,34 & 1,69 & 0 & 40 & 0 & 0 & 0 & 0 & 20 & 0 & 0 \\
\hline $2 / 2$ & 29 & 28 & 196,1 & 10,65 & 21,23 & 0,39 & 2,53 & 3,35 & 0 & 0 & 80 & 0 & 2260 & 0 & 100 & 0 & 100 \\
\hline $9 / 2$ & 28 & 35 & 147,4 & 8,99 & 5,75 & 0,20 & 0,79 & 1,97 & 0 & 0 & 80 & 0 & 0 & 0 & 40 & 20 & 40 \\
\hline $18 / 2$ & 26 & 31 & 259,4 & 11,17 & 11,48 & 0,17 & 0,97 & 1,71 & 0 & 20 & 60 & 0 & 40 & 20 & 40 & 0 & 0 \\
\hline $23 / 2$ & 27 & 32 & 389,0 & 8,10 & 28,32 & 0,15 & 1,90 & 2,72 & 0 & 20 & 0 & 0 & 20 & 0 & 60 & 20 & 20 \\
\hline $3 / 3$ & 28 & 25 & 373,6 & 9,60 & 36,71 & 0,09 & 5,96 & 2,92 & 0 & 0 & 0 & 0 & 0 & 0 & 0 & 100 & 0 \\
\hline $10 / 3$ & 29 & 32 & 184,3 & 8,10 & 33,44 & 0,13 & 4,97 & 1,28 & 0 & 0 & 0 & 0 & 0 & 0 & 0 & 80 & 120 \\
\hline $16 / 3$ & 27 & 35 & 150,2 & 10,96 & 22,42 & 0,31 & 1,37 & 2,54 & 0 & 0 & 0 & 0 & 0 & 40 & 20 & 40 & 60 \\
\hline $23 / 3$ & 27 & 34 & 144,7 & 8,68 & 13,59 & 0,51 & 1,03 & 1,31 & 0 & 80 & 0 & 20 & 0 & 40 & 0 & 0 & 0 \\
\hline $6 / 4$ & 26 & 28 & 178,4 & 12,66 & 54,12 & 0,51 & 2,26 & 1,53 & 20 & 0 & 0 & 0 & 0 & 0 & 20 & 20 & 20 \\
\hline $13 / 4$ & 23 & 35 & 175,5 & 9,33 & 17,17 & 0,69 & 5,09 & 1,56 & 0 & 0 & 0 & 0 & 0 & 0 & 20 & 0 & 20 \\
\hline $20 / 4$ & 22 & 35 & 279,6 & 6,85 & 12,43 & 0,52 & 1,97 & 2,33 & 0 & 0 & 0 & 0 & 0 & 0 & 0 & 0 & 0 \\
\hline $28 / 4$ & 22 & 34 & 144,7 & 11,08 & 46,52 & 0,27 & 3,31 & 2,80 & 0 & 40 & 0 & 0 & 0 & 0 & 40 & 100 & 60 \\
\hline $5 / 5$ & 23 & 36 & 118,4 & 8,13 & 17,21 & 0,24 & 1,63 & 1,37 & 0 & 0 & 40 & 0 & 40 & 0 & 60 & 200 & 60 \\
\hline $12 / 5$ & 22 & 35 & 123,6 & 8,39 & 12,52 & 0,49 & 0,95 & 2,51 & 0 & 0 & 0 & 0 & 20 & 0 & 20 & 0 & 0 \\
\hline $19 / 5$ & 22 & 36 & 118,4 & 9,35 & 9,24 & 0,53 & 0,85 & 0,83 & 0 & 0 & 0 & 0 & 20 & 0 & 0 & 0 & 20 \\
\hline $26 / 5$ & 20 & 35 & 98,6 & 9,67 & 14,13 & 0,57 & 1,84 & 1,37 & 0 & 40 & 0 & 0 & 0 & 40 & 40 & 0 & 0 \\
\hline $2 / 6$ & 18 & 35 & 128,8 & 8,37 & 28,17 & 0,78 & 2,61 & 0,95 & 0 & 0 & 0 & 0 & 0 & 20 & 0 & 0 & 40 \\
\hline $8 / 6$ & 18 & 31 & 103,5 & 10,20 & 31,19 & 0,75 & 3,68 & 0,96 & 60 & 0 & 0 & 0 & 0 & 100 & 20 & 0 & 60 \\
\hline $16 / 6$ & 17 & 34 & 155,7 & 7,46 & 17,72 & 0,77 & 2,30 & 1,45 & 0 & 0 & 0 & 0 & 0 & 220 & 0 & 0 & 0 \\
\hline $29 / 6$ & 19 & 31 & 139,3 & 7,35 & 22,80 & 0,82 & 2,54 & 2,93 & 40 & 0 & 0 & 0 & 0 & 20 & 0 & 0 & 60 \\
\hline Max & 29 & 36 & 388,95 & 12,66 & 54,12 & 0,82 & 6,34 & 3,98 & & & & & & & & & \\
\hline Min & 17 & 25 & 98,60 & 6,85 & 5,75 & 0,09 & 0,79 & 0,83 & & & & & & & & & \\
\hline$x$ & 23,92 & 32,71 & 191,01 & 9,30 & 22,47 & 0,43 & 2,43 & 1,97 & & & & & & & & & \\
\hline s & 3,63 & 3,07 & 84,18 & 1,51 & 13,44 & 0,22 & 1,65 & 0,86 & & & & & & & & & \\
\hline CV & 15,20 & 9,39 & 44,07 & 16,28 & 59,82 & 51,48 & 67,71 & 43,43 & & & & & & & & & \\
\hline $\mathrm{N}$ & 24 & 24 & 24 & 23 & 24 & 24 & 24 & 24 & & & & & & & & & \\
\hline EP & 0,74 & 0,63 & 17,18 & 0,32 & 2,74 & 0,05 & 0,34 & 0,18 & & & & & & & & & \\
\hline
\end{tabular}


= concentração em $\mathrm{ml}(1000 \mathrm{ml}) ; \mathrm{Vf}=$ volume do filtrado em $\mathrm{ml}(200)$

Os valores de vazão do rio Itajaí - Açu foram cedidos pelo professor Carlos Augusto Schettini, do Laboratório de Oceanografia Física (CTTMar). Estes dados foram obtidos no município de llhota/SC, através de determinação indireta de vários parâmetros hidrológicos, sendo a vazão expressa em $\mathrm{m}^{3} \cdot \mathrm{s}^{-1}$. As variáveis salinidade e temperatura, foram medidas conjuntamente no local da coleta, por refratometria e por termômetro de mercúrio, respectivamente. Os dados obtidos foram analisados através de visualização em figuras de linhas e medidas de tendência central. Para evidenciar as possíveis relações entre as variáveis, foi realizada uma análise de componentes principais (ACP).

\section{RESULTADOS E DISCUSSÃO}

A temperatura da água variou entre $29^{\circ} \mathrm{C}$ e $17^{\circ} \mathrm{C}$ (Figura 2), sendo que no verão ela esteve mais alta que no outono. Este padrão de distribuição é típico de nossa região com predomínio de clima subtropical, como verificado em outros trabalhos (Rörig, et al.,1998; Pereira et al. 1998). A salinidade variou entre $36 \mathrm{e}$ 25 (Figura 2), valores baixos indicam a influência da descarga do Rio Itajaí-Açu ao ponto amostral em Cabeçudas. Esta relação é observada principalmente em períodos de maior descarga no estuário. Os valores máximos e mínimos de descarga foram de $389 \mathrm{~m}^{3} . \mathrm{s}^{-1} \mathrm{e}$ $98,6 \mathrm{~m}^{3} \cdot \mathrm{s}^{-1}$, respectivamente (Figura 3 ). Os dados da descarga do rio Itajaí-Açu estiveram em geral, abaixo da média descrita por Schettini \& Carvalho (1998) que foi de $318 \pm 394 \mathrm{~m}^{3} . \mathrm{s}^{-1}$. Assim, espera-se que valores de salinidade ainda menores sejam encontrados no ponto amostral. A baixa descarga esteve associada a baixa pluviosidade na região de drenagem do Rio Itajaí-Açu durante o período amostral. Os nutrientes inorgânicos dissolvidos tiveram grande variação durante os seis meses de amostragem. Os máximos de nitrato, amônio, silício e fosfato foram de $6,34 \mu \mathrm{mol} . \mathrm{I}^{-1}$, $12,66 \mu \mathrm{mol} . \mathrm{I}^{-1}, 54,12 \mu \mathrm{mol}^{.} \mathrm{I}^{-1}$ e $0,82 \mu \mathrm{mol}^{-\mathrm{I}^{-1}}$, respectivamente (Figura 4), enquanto os mínimos foram de $0,76 \mu \mathrm{mol} . \mathrm{I}^{-1}, \quad 6,85 \mu \mathrm{mol} . \mathrm{I}^{-1}$, $5,75 \mu \mathrm{mol}^{-\mathrm{I}^{-1}}$ e $0,09 \mu \mathrm{mol}^{-\mathrm{I}^{-1}}$. Estes valores estão próximos dos limites máximos descritos por Pereira et al. (1998), encontrados na Enseada de Armação do Itapocoróy/Penha, ao norte da praia de Cabeçudas. O nitrato apresentou períodos de máximos e mínimos bem definidos durante os meses de temperaturas mais elevadas, sendo que em temperaturas mais frias não ocorreu um padrão definido. O silício teve o mesmo comportamento do nitrato. $\mathrm{O}$ amônio oscilou pouco sua concentração, se comparado ao valor da média, que foi de $9,30 \mu \mathrm{mol}^{-\mathrm{I}^{-1}}$. Períodos de concentração mínima de fosfato estiveram associados a altas temperaturas e períodos de concentração má-

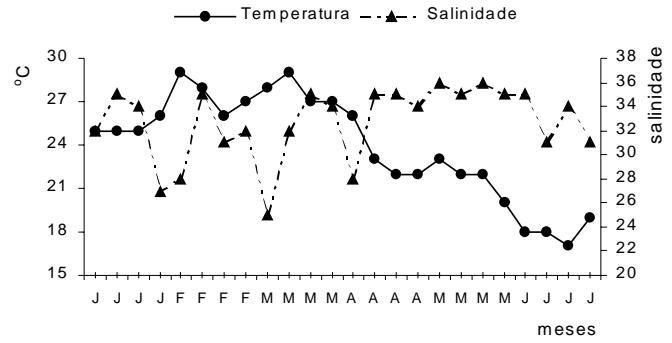

Figura 2 - Temperatura e salinidade da água junto ao late Club entre os dias 6/1/99 e 29/6/99.

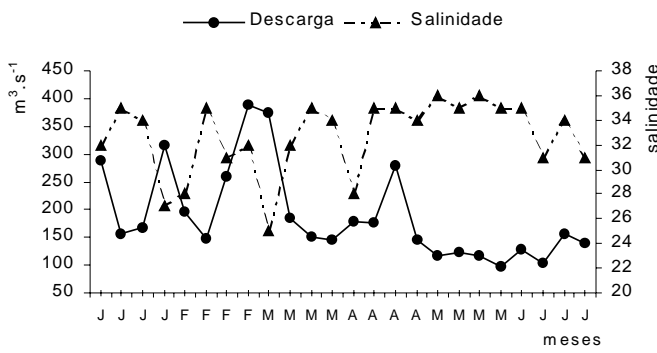

Figura 3 - Descarga do Rio Itajaí-Açú medidos no município de Ilhota entre os dias 6/1/99 e 29/6/99 e salinidade no ponto amostral. 
xima, à baixas temperaturas. Em períodos de alta descarga do rio Itajaí-Açu e baixa salinidade, as concentrações de nitrato e silício foram altas, sendo o contrário também verificado. Geralmente em ambientes costeiros, dominados pelo aporte de água doce, a variação de nitrato acompanha a de silicatos, característicos da drenagem continental. O máximo e mínimo de cla a foram de 3,97 $\mu \mathrm{g} . \mathrm{I}^{-1}$ (12/ $1 / 99)$ e $0,83 \mu \mathrm{g} . \mathrm{l}^{-1}(19 / 5 / 99)$, respectivamente (Figura 5). Foi observada uma grande variação ao longo do período amostral, não sendo possível a identificação de um padrão de distribuição. O máximo valor de cla a no dia 12/1/99, esteve associado ao aparecimento da microalga Trichodesmium sp., que aparece na costa catarinense nos meses de novembro à abril (Rörig et al., 1998). Estes valores de cla a estão dentro dos valores medidos por Proença \& Schettini (1998), indicando um ambiente rico em biomassa porém, não eutrófico.

As espécies de dinoflagelados encontrados durante o período amostral foram quatro: $D$. acuminata, $D$. caudata, $D$. rotundata e Dinophysis spp. Este resultado é semelhante
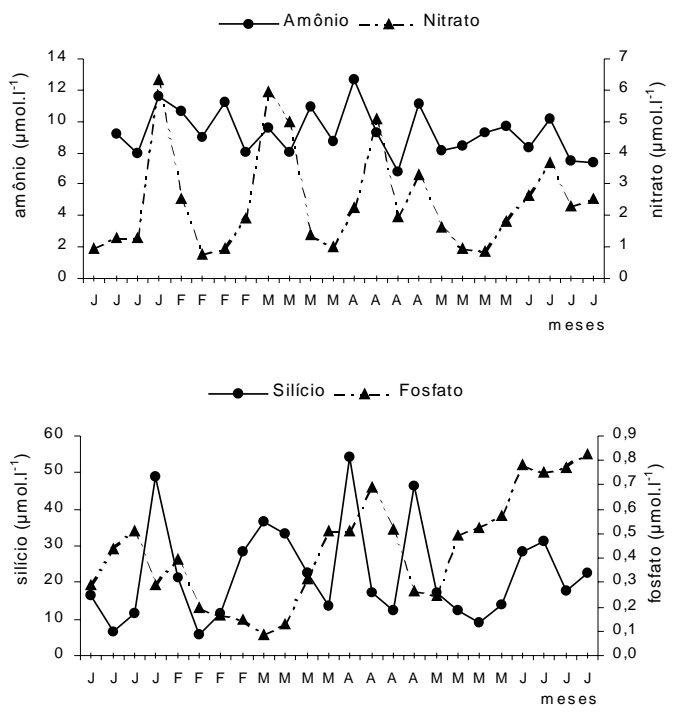

Figura 4 - Nutrientes inorgânicos, silício, fosfato, nitrato e amônio em $\mu \mathrm{mol}^{.} \mathrm{I}^{-1}$, entre os dias 6/1/99 e 29/6/99, junto ao trapiche do late Club. ao de Rörig et al. (1998), que encontraram na Enseada de Armação do Itapocoróy $D$. acuminata e $D$. caudata. Porém, em nosso litoral já foram observadas seis espécies de Dinophysis: D. caudata, D. ovum, D. acuminata, $D$. tripos, D. acuta, e D. rotundata (Rörig, L. R., CTTMar/UNIVALI, com. pes.). Estes resultados indicam que nem todas as espécies de Dinophysis aparecem em uma mesma amostra ou área de estudo, durante o período de janeiro a junho. Em geral, a abundância de Dinophysis foi baixa, sendo encontrado o máximo de 80 cel. I $^{-1}$ para $D$. caudata, no dia 2/2/ 99 e 9/2/99 e 80cel. I $^{-1}$ para $D$. rotundata no dia 23/3/99 (Figura 6). Estes valores são muito baixos, se comparado a alta abundância observada em Rörig et al. (1998), que descrevem a concentração de 2206 cel..$^{-1}$ de $D$. caudata, na Enseada de Armação do Itapocoróy, no final do inverno e início da primavera, no dia 7/9/ 96. Os valores de abundância encontrados na praia de Cabeçudas durante este trabalho são inferiores aos encontrados na Enseada de Armação do Itapocoróy. Por outro lado, no referido trabalho, não foram encontradas Dinophysis entre os meses de janeiro à julho. Estes resultados indicam que a distribuição e ocorrência de Dinophysis na região não está associada diretamente a época do ano. Em um trabalho realizado na costa Atlântica da França, Delmas et al. (1992) encontraram altas densidades de Dinophysis em profundidades entre 5 à $20 \mathrm{~m}$,

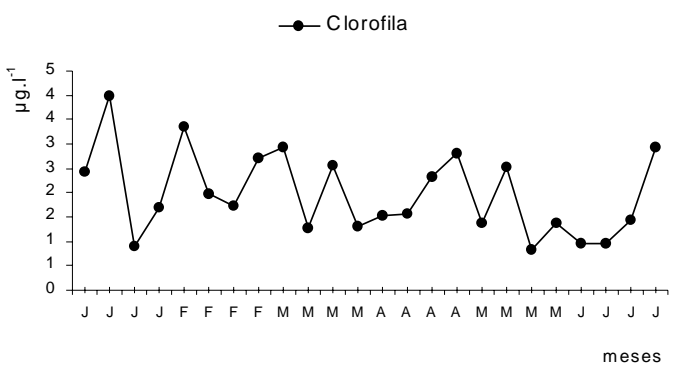

Figura 5 - Variação da clorofila a entre os dias 6/1/99 e 29/6/99 junto ao late Club em Cabeçudas. 
devido provavelmente a capacidade de locomoção das Dinophysis através dos flagelos. Maestrini (1998) descreve ainda que as espécies de Dinophysis têm a capacidade de realizar migrações verticais. No presente trabalho, as amostragens foram realizadas a profundidades de cerca de $2 \mathrm{~m}$, em uma camada rasa, não evidenciando altas concentrações de Dinophysis. Em função da salinidade, Delmas et al. (1992), mostram que ocorre um pequeno aumento na abundância de Dinophysis com o aumento da salinidade, o que não se verifica na praia de Cabeçudas, onde Dinophysis spp. ocorrem em períodos tanto de baixa como de alta salinidade.

Em relação aos nutrientes inorgânicos dissolvidos, Johansson et al. (1996) não encontraram uma relação com a abundância de Dinophysis. Delmas et al. (1992), também não encontraram nenhuma correlação entre as espécies de Dinophysis e a concentração de nutrientes. Aparentemente não existe relação direta entre o aparecimento de Dinophysis e a concentração de nutrientes. Hews et al. (1998), evidenciaram a presença de pigmentos fotossintéticos de outros grupos de microalgas em Dinophysis spp., sugerindo desta forma que as espécies de Dinophysis realizam fagotrofia (endossimbiose).

A produção de toxinas por Dinophysis não está elucidada totalmente. Johansson et al., (1996), sugerem que a deficiência de nitrogênio no ambiente marinho aumentaria a pro-

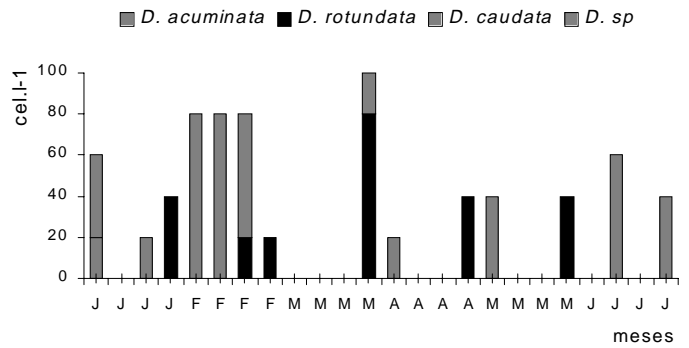

Figura 6 - Abundância das espécies de Dinophysis junto ao late Club durante o período de 6/1/99 e 29/6/99. Espaços vazios indicam ausência de Dinophysis no dia da amostragem. dução das toxinas de DSP em Dinophysis acuminata, sendo o inverso observado para a produção de toxinas em Dinophysis acuta. Já, Flynn \& Flynn (1995), sugerem que as toxinas sejam metabólitos secundários dependentes do metabolismo primário, originados em situação de estresse. Um importante aspecto relacionado aos nutrientes inorgânicos dissolvidos é a variação da concentração de toxinas ocasionada pela variação na concentração dos nutrientes. Johansson et al. (1996) relatam, que a carência ou aumento da concentração de nutrientes causa variação na concentração das toxinas $\mathrm{AO}$ em cada célula. Os autores citam ainda que o máximo de $A O$ encontrado por célula foi de 40pg. Trabalhos realizados em nossa região, descrevem a concentração de $7,42 \mathrm{pg}$ de $\mathrm{AO}$ por célula em $D$. acuminata (Proença, et al., 1998). Estes resultados mostram o potencial de variação da concentração de $A O$ em Dinophysis em diferentes regiões de estudo. As análises de correlação simples apresentaram resultados semelhantes aos da análise de componentes principais. A visualização das interações entre as variáveis pode ser observada na figura 7 , da análise de componentes principais. Os dois fatores analisados representaram $87,69 \%$ da variância total explicada (Tabela 2), 34,95 para o fator $1 \mathrm{e}$ 52,74 para o fator 2 . Esta porcentagem foi con-

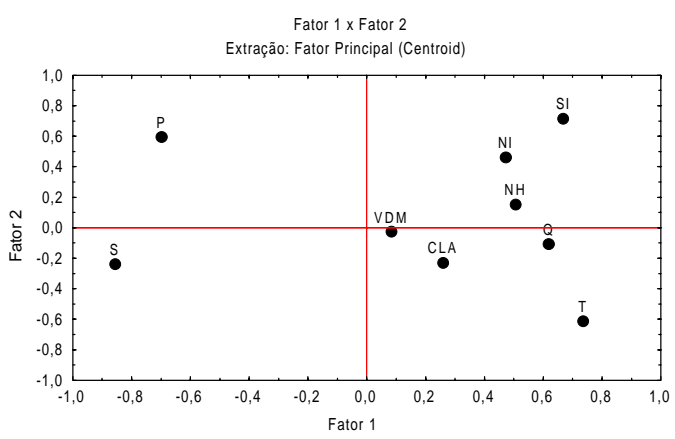

Figura 7 - Plotagem das variáveis em relação aos dois eixos extraídos da análise da ACP. Temperatura (T), salinidade $(\mathrm{S})$, descarga $(\mathrm{Q})$, amônio $(\mathrm{NH})$, silício $(\mathrm{SI})$, fosfato $(\mathrm{P})$, nitrato $(\mathrm{NI})$, clorofila a (cla), e somatório de Dinophysis spp. (VDM). 
siderada significativa, tendo em vista a pouca quantidade de amostras. $\mathrm{Na}$ análise das componentes, a temperatura teve relação inversa com a salinidade, fosfato, silício e nitrato. A salinidade teve correlação indireta com todas as outras componentes, dominando o eixo do fator 1 negativo. Este resultado evidencia a entrada de uma massa de água mais fria, com características físico-químicas diferentes das águas estuarinas. O fosfato teve o mesmo comportamento da salinidade, porém, dominou o eixo 2 com valores positivos. O silício, nitrato e amônio estão conjuntamente agrupados, mostrando uma forte relação entre estes três nutrientes. Esta relação sugere que estes três nutrientes são aportados conjuntamente pelo rio Itajaí-Açu. Os valores de cla a não demonstram relações claras entre as componentes da análise. O mesmo se verifica para VDM (somatório de Dinophysis spp.), que está muito próximo da intersecção dos eixos, indicando pouca significância. Desta forma, estes dois componentes, podem ser dominados por outros fatores que não foram envolvidos neste trabalho.

\section{CONSIDERAÇÕES FINAIS E CONCLUSÕES}

Neste trabalho, os valores potenciais de VDM (somatório de Dinophysis spp.), não apre- sentam nenhuma correlação com as variáveis analisadas, sugerindo que 0 aparecimento das espécies de Dinophysis não depende destes fatores, ao menos nos períodos de verão e outono. Os valores de temperatura e salinidade, entre os meses de janeiro a junho, também não ocasionariam o aparecimento das espécies de Dinophysis. Vários trabalhos indicam o aparecimento de Dinophysis em abundância no inverno. Desta forma, estes resultados sugerem que as espécies de Dinophysis apresentam variações anuais e interanuais de distribuição. Das seis espécies citadas para a região, apenas três ocorreram na praia de Cabeçudas, sendo: $D$. acuminata, $D$. caudata e $D$. rotundata. A abundância das espécies de Dinophysis também foi baixa, não ultrapassando as $80 \mathrm{cel}^{-\mathrm{I}^{-1}}$. Apesar da baixa diversidade e abundância das espécies de Dinophysis, estas ocorreram em mais de $50 \%$ das amostras analisadas sugerindo que as Dinophysis estão presentes, porém as condições ambientais não favorecem o seu crescimento. Outros organismos produtores ou potencialmente produtores de toxinas lipossolúveis ou paralisantes também foram observados durante o período amostral. Dentre os mais importantes estão: G. catenatum, Trichodesmium sp., Prorocentrum sp. e Ceratium sp. Do ponto de vista da baixa abundância das espécies de Dinophysis, a praia de Cabeçudas proporcio-

Tabela 2 - Resultado da análise de componentes principais mostrando o peso das variáveis analisadas nos dois fatores extraídos e o peso de cada fator. $\mathrm{O}\left({ }^{*}\right)$ indica a significância da análise.

\begin{tabular}{|c|c|c|}
\hline Análise de Fator & \multicolumn{2}{|c|}{$\begin{array}{c}\text { Extração: Fator principal (Centróide) } \\
\text { Significativo }>0,70000\end{array}$} \\
\hline Variável & Fator $1(34,95 \%)$ & Fator $2(52,74)$ \\
\hline Temperatura (T) & ${ }^{*} 0,735$ & $-0,612$ \\
\hline Salinidade (S) & ${ }^{*}-0,855$ & $-0,239$ \\
\hline Descarga (Q) & 0,619 & $-0,106$ \\
\hline Amônio (NH) & 0,507 & 0,151 \\
\hline Silício (SI) & 0,668 & ${ }^{*} 0,716$ \\
\hline Fosfato $(P)$ & $-0,697$ & 0,595 \\
\hline Nitrato (NI) & 0,473 & 0,460 \\
\hline Clorofila a (CLA) & 0,259 & $-0,228$ \\
\hline Somatório Dinophysis (VDM) & 0,084 & $-0,026$ \\
\hline Explo. Variável & 3,146 & 1,600 \\
\hline Proporção total & 0,349 & 0,177 \\
\hline
\end{tabular}


naria um local apropriado para o cultivo e extração de moluscos marinhos, uma vez que seria em geral, livre de toxinas causadoras de DSP. Porém, ocorre a presença de outros organismos tóxicos, além de outros tipos potenciais de contaminação. Neste caso, a extração ou cultivo de moluscos na área deveria ser monitorada frente aos contaminantes potenciais.

\section{REFERÊNCIAS BIBLIOGRÁFICAS}

Andersen, N.P. 1996. Design and implementation of some harmful algal monitoring systems. IOC Technical Series n. 44, UNESCO.

Baumgarten, M.Z.; Rocha, J.M. \& L.F.H. Niencheski. 1996. Manual de análises em oceanografia química. Ed. Rio Grande:Furg.

Cardoso, L.S. 1993. Dinoflagelados da llha do Arvoredo e da Praia de Pontal das Canas SC, Brasil (setembro de 1991 a fevereiro de 1992): Considerações taxonômicas e ecológicas. Dissertação de Mestrado, Porto Alegre, UFRGS.

Carreto. J.I. 1991. Marés vermelhas. Ciência Hoje. 13:52-60.

Delmas, D.; Herbland, A. \& S.Y. Maestrini. 1992. Environmental conditions which lead to increase in cell density of the toxic dinoflagellates Dinophysis spp. in nutrientrich and nutrient-poor waters of the French Atlantic coast. Mar. Ecol. Prog. Ser. 89:5361.

Flynn, J. K. \& K. Flynn. 1995. Dinoflagellate physiology: nutrient stress and toxicity.p. 541-550. In: P. Lassus; G. Arzul; E. ErardLe-Denn \& P. Gentien [Eds.], Harmful Marine Algal Blooms. Lavoisier.

Hallegraeff, G.M.; Anderson, D.M. \& A.D. Cembella. 1995. Manual on Harmful Marine Microalgae. IOC Manuals and Guides, n. 33, UNESCO.

Hallegraeff, G.M. 1993. A review of harmful algal blooms and their apparent global increase. Phycologia, 32(2):79-99.
Hews, C.D.; Mitchell, B.G. \& T.A. Moisan. 1998. The phycobilin signatures of chloroplasts from three dinoflagellate species: a microanalytical study of Dinophysis caudata, $D$. fortii, and $D$. acuminata (Dinophysiales, Dinophyceae). Journal Phycol. 34:945-951.

Intergovernmental Oceanographic Commission.1995. Manual on harmful marine microalgae. UNESCO.

Johansson, N.; Granéli, E. \& T. Yasumoto. 1996. Toxin prodution by Dinophysis acuminata and $D$. acuta cells growm under nutrient sufficient and deficient conditions p. 277280. In: T. Yasumoto; T. Oshima \& Y. Fukuyo [Eds.], Harmful and Toxic Algal Blooms. IOC of UNESCO.

Kat, M. 1979. The occurrence of Prorocentrum species and coincidental gastrointestinal illnes of mussel consumers p. 215-220. In: D. Taylor \& H.H. Seiger [Eds.], Toxic dinoflagellate blooms. Amsterdam:Elsevier.

Larsen, J. \& O. Moestrup. 1989. Guide to toxic and potentially toxic marine algae. University of Copenhagen. The Fish Inspection Service, Ministry of Fisheries, Denmark.

Maestrini, S.Y. 1998. Dynamics and ecophysiology of Dinophysis spp. p. 243265. In: D.M. Anderson; A.D. Cembella \& G.M. Hallegraeff [Eds], Physiological Ecology of Harmful Algal Blooms. SpringerVerlag.

Mantoura, R.F.C.; Barlow, R.G. \& E.J. Head. 1997. Simple isocratic HPLC methods for chlorophylls and their degradation products. p. 307-226. In: S.W. Jeffrey; R.F. Mantoura \& S.W. Wright [Eds.], Phytoplankton pigments in oceanography. UNESCO.

Margalef, R. 1983. Limnologia. Omega.

Pereira Fo, J.; Oliveira, U.C. \& G.C. Manzoni. 1998. O uso do Bell Jar na avaliação do metabolismo bentônico na Armação do Itapocoróy: resultados preliminares.. Notas Técnicas FACIMAR, 2:81-92.

Proença, L.A., Schmitt, F.; Costa, T. \& L. Rörig. 1998. Produção de ácido okadaico por 
Dinophysis acuminata em Santa Catarina. p. 69. Encontro Brasileiro de Ecotoxicologia, 5o (5 ECOTOX), Colóquio Brasileiro de Algas Nocivas, 1, (1 COBAN).

Proença, L.A. \& C.A.F. Schettini. 1998. Effect of shellfish culture on phytodetritos vertical fluxes in tropical waters - southern Brazil. Rev. Bras. Oceanogr. 46(2):125-133.

Proença, L.A.; Schmitt, F.; Costa, T. \& L. Rörig. 1998. Just a diarrhea? Evidence of diarrhetic shellfish poisoning in Santa Catarina, Brazil. Ciência e Cultura. 50(6):458-462.

Proença, L.A.; Rörig, L.; Barreiros, M.A. \& N. Lagos. 1996. First occurrence of okadaic acid, a diarrhetic shelfish toxin in cultured mussels in the brasilian coast. In: IV Congres Latino-Americano de Ficologia, Caxambú.

Rörig, L.R.; Guimarães, S.G.; Lugli, D. \& L.A. Proença. 1998. Monitorização de microalgas planctônicas potencialmente tóxicas na área de maricultura da Enseada de Armação do Itapocoróy-Penha/SC. Notas Técnicas da FACIMAR. 2:71-79.

Schettini, C.A.F. \& J.L.B. Carvalho. 1998. Hidrodinâmica e distribuição de sólidos em suspensão no estuário do Rio Itajaí - Açu (Relatório). Notas Técnicas da FACIMAR. 2:131-140.

Utermohl, H. 1958. Zur vervollkommung der quantitativen phytoplankton methodik. Mitt. Int. Ver. Limnol. 9:1-38.

Yasumoto, T.; Oshima, Y.; Sugawara, W.; Fukuyo, Y. \& H. Ogury. 1978. Identification of Dinophysis fortii as the causative organism of diarrhetic shellfish poisoning. Bull. Jap. Soc. Sc. Fish. 46:1405-1411.

Zenebon, O. \& N.P. Pregnolatto. 1992. Memórias técnico-científicas da divisão de bromatología e química p. 173-198. In: J.L.F. Antunes. Instituto Adolfo Lutz - 100 Anos de Saúde Pública Secretaria de Estado da Saúde - SP. Instituto Adolfo Lutz. 